\title{
Individual Differences in the Allocation of Visual Attention during Navigation
}

Mikayla Keller, The University of Western Ontario

Supervisor: Dr. Jennifer E. Sutton, The University of Western Ontario

A thesis submitted in partial fulfillment of the requirements for the Master of Science degree in Psychology

(c) Mikayla Keller 2017

Follow this and additional works at: https://ir.lib.uwo.ca/etd

Part of the Cognition and Perception Commons, and the Cognitive Psychology Commons

\section{Recommended Citation}

Keller, Mikayla, "Individual Differences in the Allocation of Visual Attention during Navigation" (2017). Electronic Thesis and Dissertation Repository. 4693.

https://ir.lib.uwo.ca/etd/4693

This Dissertation/Thesis is brought to you for free and open access by Scholarship@Western. It has been accepted for inclusion in Electronic Thesis and Dissertation Repository by an authorized administrator of Scholarship@Western. For more information, please contact wlswadmin@uwo.ca. 


\begin{abstract}
There are large individual differences in the ability to create an accurate mental representation (i.e., a cognitive map) of a novel environment, yet the factors underlying cognitive map accuracy remain unclear. Given the roles that landmarks and cognitive map accuracy play in successful navigation, the current study examined whether differences in the landmarks that individuals look at while navigating are related to differences in cognitive map accuracy. Participants completed a battery of spatial tests: some that assessed spatial skills prior to a navigation task, and others that tested memory for the environment following exploration of a virtual world. Results indicated that individuals with inaccurate maps had weak perspective-taking abilities, struggled to create shortcuts, and remembered fewer landmarks despite having looked at target buildings and objects in the environment for the same duration as individuals with accurate cognitive maps. These findings suggest that memory capabilities underlie differences in cognitive map accuracy.
\end{abstract}

\title{
Keywords
}

Cognitive map, navigation, virtual reality, eye tracking, memory, attention, individual differences 


\section{Acknowledgements}

The completion of this thesis would not have been possible without the many people that have supported me.

Thank you to my advisor, Dr. Jennifer Sutton, for her continuous encouragement, praise, inspiration, and support. Her guidance has been invaluable to the completion of this thesis, and without her enthusiasm and the opportunity to get involved in this research I would not be where I am today.

I would also like to thank Chantelle Cocquyt for her incredible work as a research assistant on this project. Her attention to detail and commitment to the project is greatly appreciated.

I am grateful to have had the opportunity to work with all past and present members of the Spatial Cognition Laboratory. Thank you for your support and feedback on presentation rehearsals.

Thank you to Dr. Marc Joanisse for his expertise and being of assistance when I had questions in regards to retrofitting our existing eye tracking setup for dynamic eye tracking.

Thanks to SR Research Ltd. for their dynamic eye tracking beta software and technical support.

To my family and friends: Your incredible support and encouragement has, no doubt, helped me through these past two years. Most importantly, thank you Mom, Dad, and my sister Alyssa. I am forever grateful for your love, advice, and support. Thank you for always being there for me along the way. 


\section{Table of Contents}

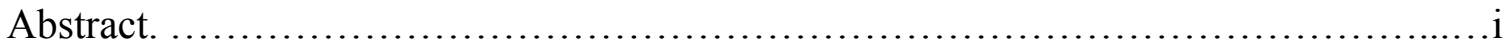

Acknowledgements. ..........................................................

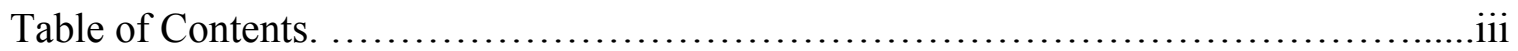

List of Tables. ...............................................................

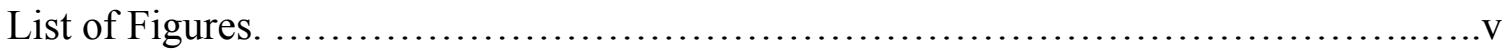

List of Appendices. ........................................................ vi

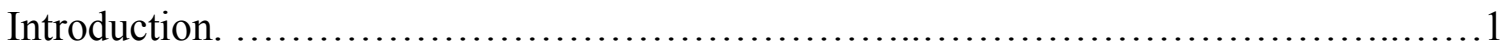

Method. ..................................................................... 11

Participants. ...................................................... 11

Materials and Procedure. ..................................................11

Analysis of Eye Movement Data. ...................................... 17

Statistical Analyses. ............................................... 18

Results. ......................................................................

Full Sample. .......................................................19

Participant Classification. ..........................................22

Comparisons of Individuals with Accurate and Inaccurate Cognitive Maps. ......23

Discussion. ............................................................. 32

References. ............................................................40

Appendix A: Ethics Approval. ...............................................44

Curriculum Vitae. ........................................................45 


\section{List of Tables}

Table 1: Correlations between age, sex, mobility, video game use, SBSOD performance,

SOT performance, and measures of spatial ability pertaining to Silcton. .................21 


\section{List of Figures}

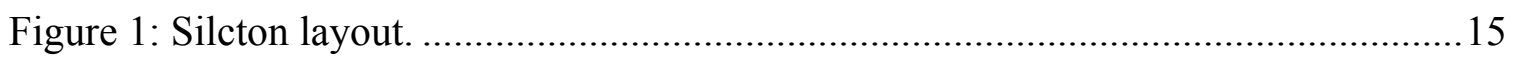

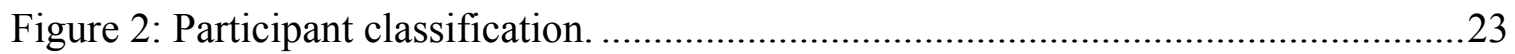

Figure 3: Composite cognitive map error scores.......................................................25

Figure 4: Performance on assessments of spatial ability and gaming frequency. ............26

Figure 5: Attention to Silcton item categories.......................................................29

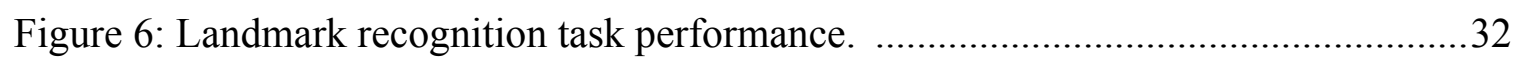




\section{List of Appendices}

Appendix A: Ethics Approval. ..............................................44 


\section{Introduction}

Navigation is a task that is central to the lives of humans and animals. It is the way in which individuals find their way around, whether it is for the purpose of travelling to school, finding food, or making it to work on time. One way that individuals find their way around is by using visual landmarks. Landmarks can generally be defined as a feature of the environment that serves as a navigational aid, either in understanding the location of oneself in an environment, assisting in finding one's way to a goal destination, or identifying the location of other landmarks through association (e.g., Gallistel, 1990; Golledge, 1999; Presson \& Montello, 1988; Siegel \& White, 1975; Sorrows \& Hirtle, 1999). Individuals can think about landmarks and other objects around them in one of two ways. The first, known as an egocentric reference system, involves developing knowledge of the locations of landmarks relative to one's own position. An egocentric reference system leads to the development of route knowledge by linking actions to landmarks, such as turn left at the gas station and right at the library (Aguirre \& D’Esposito, 1999; Gallistel, 1990). On the other hand, a strong allocentric reference system involves a good understanding of the relationship between landmarks (Aguirre \& D’Esposito, 1999; Gallistel, 1990). The allocentric reference system leads to a richer understanding of the layout of the environment, resulting in a mental representation similar to a map or a cognitive map (Gallistel, 1990).

A cognitive map is a configurational mental representation, akin to a physical map, of the layout of a large-scale area (Siegel \& White, 1975; Tolman, 1948; Tolman, Ritchie, \& Kalish, 1946). It is orientation-independent, meaning the perspective of the representation is flexible and not tied to the individual's current viewpoint, and it can 
include landmarks, roads, and other objects. Unlike route knowledge, a cognitive map facilitates navigation because it provides a global representation of the environment, enabling an individual to solve various navigation problems, such as identifying one's current location, identifying a goal destination, and judging distances and directions to and from locations. Having an accurate cognitive map also allows an individual to take novel shortcuts and detour routes (O'Keefe \& Nadel, 1978; Tolman, 1948; Tolman et al., 1946). For instance, Tolman (1948) found evidence of cognitive maps in rats after observing that rats were able to take shortcuts to locations of food in a maze using paths that they had not previously travelled. In addition, O'Keefe and Nadel proposed that the ability to take novel shortcuts distinguished rats that navigated via a cognitive map from rats that navigated from one landmark to another along a route. The ability to take novel shortcuts has since become a defining feature of representing space as an accurate cognitive map (Bennet, 1996), because it requires inferences about where a novel route will lead based only on previous encounters with other segments of the environment. In particular, previous work with humans has evaluated cognitive map accuracy by testing the ability to switch from following a pre-determined learned route to identifying novel shortcuts to get to a goal location (Foo, Warren, Duchon, \& Tarr, 2005; Harris \& Wolbers, 2014). Estimating the direction from one landmark to another has also been used as a measure of cognitive map accuracy as it relies on similar processing as the ability to take novel shortcuts (Hegarty, Montello, Richardson, Ishikawa, \& Lovelace, 2006; Ishikawa \& Montello, 2006; Weisberg \& Newcombe, 2016; Weisberg, Schinazi, Newcombe, Shipley, \& Epstein, 2014). 
Two theories attempt to explain how people develop a cognitive map during direct exposure to an unfamiliar environment. Siegel and White (1975) proposed a stagelike framework and suggested that individuals initially acquire identity information about the landmarks in the environment, such as their names and features. In the next stage, route information is acquired by chaining landmarks together. In the third and final stage of the framework, cognitive map knowledge (also sometimes referred to as survey knowledge), such as the locations of and distances between landmarks, is learned. In order to reach the third stage, the individual routes from the second stage must be scaled and interrelated to understand how they connect to each other and form a global representation of the environment. Siegel and White proposed that this third stage is the most difficult, and not everyone is capable of achieving it. Studies in the 1970's and 1980's largely supported the stage-like framework (Allen, Kirasic, Siegel, \& Herman, 1979; Cohen \& Schuepfer, 1980; Cousins, Siegel, \& Maxwell, 1983; Curtis, Siegel, \& Furlong, 1981; Evans, Marrero, \& Butler, 1981; Hazen, Lockman, \& Pick, 1978). Later, however, Montello (1998) noted that some individuals appeared to acquire cognitive map knowledge upon initial exposure to an environment. For example, some participants were able to take new shortcut routes to get from one landmark to another after only brief exposure to a specific set of paths (e.g., Klatzky et al., 1990; Landau, Spelke, \& Gleitman, 1984; Loomis et al., 1993). Thus, Montello proposed that the creation of mental spatial representations of the environment were better characterized as a continuous framework, where landmark, route, and cognitive map knowledge could be acquired simultaneously, rather than in stages. In particular, he argued that cognitive map knowledge, in addition to landmark and route knowledge, could be acquired upon first exposure to an 
environment, and increased exposure to the environment could result in more accurate knowledge for some people but not others. Both frameworks highlight the potential for individual differences in the ability to acquire spatial knowledge of the environment. Individual differences in cognitive map accuracy were confirmed in a study conducted by Ishikawa and Montello (2006). In the study, researchers drove participants on two different routes in an unfamiliar area once per week for 10 weeks. Participants were instructed to pay attention to specific landmarks on these routes. Beginning in the fourth week, participants were also exposed to a connecting route. Following each driving session, a battery of spatial tasks was administered to determine whether participants integrated the routes into a single representation. The tasks included a pointing task where participants were required to estimate the direction from one landmark to another and involved landmarks that were either on same or different routes, and a map drawing task where participants created a map of the entire environment. The study took place in an area that was selected for its hilly landscape so that landmarks could not be seen from where a participant was positioned when they were required to estimate their directions. The ability to accurately estimate the direction of an unseen landmark suggests that an individual has an accurate cognitive map representation of the environment. Supporting Montello's (1998) theory, yet contrary to Siegel and White's (1975) theory, performance on the pointing and map drawing tasks indicated that 4 of the 24 participants acquired cognitive map knowledge upon immediate exposure to the routes, as accuracy on these tasks was above chance after the initial session. These participants continued to show improvement over the course of the study. On the other hand, some individuals showed poor initial performance and no improvement over the course of the 
10 weeks. The results indicated that forming an accurate cognitive map was easy for some people yet quite difficult for others, supporting the existence of individual differences in cognitive map accuracy.

More recently, Weisberg et al. (2014) replicated the finding of individual differences in cognitive map formation using a desktop non-immersive virtual environment (Silcton) rather than a real-world environment. Participants traversed two non-overlapping main routes and two connecting routes in Silcton. While exploring these four routes, participants were instructed to remember the names and locations of eight target buildings. After traversing the four routes, participants completed two tasks that assessed their memory of the environment including a direction estimation task and a map-building task resembling those used by Ishikawa and Montello (2006). For each trial in the direction estimation task, participants were placed back in the environment standing at a particular landmark and were required to estimate the direction of a second landmark. Trials were divided into within-route and between-route trials. Within-route trials involved estimating the direction between two buildings on the same route, providing a measure of knowledge of the directions of landmarks along a path, indicating route knowledge. Between-route trials involved estimating the direction between two buildings on different routes requiring an ability to integrate the four separate routes into a single representation, indicating cognitive map knowledge.

As in the results of Ishikawa and Montello (2006), there was considerable range in direction estimation accuracy in the study by Weisberg et al. (2014). Some participants showed both accurate route knowledge and accurate cognitive map knowledge. Others were accurate on within-route trials, but showed poor performance on between-route 
trials, and some participants showed poor performance on both within-route and betweenroute trials. Few participants showed a pattern of poor performance on within-route trials and good performance on between-route trials, which is consistent with Siegel and White's (1975) theory that route knowledge must be acquired before cognitive map knowledge. Thus, when using a virtual environment, Weisberg et al.'s results were similar to Ishikawa and Montello's indicating that there was significant variation among individuals in the ability to form an accurate cognitive map of a novel environment.

In addition to differences in the ability to create a cognitive map, non-spatial information about landmarks seems to be difficult for some individuals to remember. When performing the direction estimation task, some participants performed poorly even when the buildings they were asked to point to were on the same route in plain view (Weisberg et al., 2014). That is, some people could not estimate the direction of a building that could be clearly seen. In a subsequent study, Weisberg et al. (2016) noted that individuals with inaccurate cognitive maps also remembered fewer building names than participants with more accurate maps. Together, these findings suggest that poor cognitive map accuracy is associated with weak memory for non-spatial information about landmarks. It could be that some individuals have trouble remembering the appearances of buildings or the building names, or they are unable to link the names with the appearances and store this information in memory. Another possibility is that some individuals missed or failed to look at certain buildings while navigating. Given the connection between retaining landmark information and cognitive map accuracy, it seems important to understand how and whether landmark information is processed while navigating and how landmark information affects cognitive map accuracy. 
It is possible that, in addition to individual differences in cognitive map accuracy, there are individual differences in the features of the environment that individuals look at. One way to determine what people look at while navigating is to use a dynamic eye tracking technique. Even though eye tracking in spatial cognition research has mostly been employed in small-scale tasks, such as the mental rotation task (see Nazareth, Odean, \& Pruden, 2017 for an overview), there are a few studies where dynamic eye tracking was used in conjunction with virtual environments. For instance, Andersen, Dahmani, Konishi, and Bohbot (2012) investigated sex differences in landmark use during navigation in a maze environment by manipulating the number of landmarks available and measuring looking time to the landmarks. They found that women looked at landmarks more than men and showed poorer navigation performance when fewer landmarks were available. The sex difference in navigation performance, however, disappeared when more landmarks were present in the environment, highlighting differences in looking times to landmarks and the role that landmarks play in navigation ability.

In another study, Hamid, Stankiewicz, and Hayhoe (2010) also used a maze environment and evaluated the link between eye fixations and encoding of landmarks while navigating. After initial navigation of a novel 10-corridor virtual maze environment, landmarks that were fixated on for the least amount of time were removed for subsequent navigation trials. Removal of these landmarks produced no significant decrements in performance when participants were asked to navigate from one location to another taking the most direct route. However, when landmarks that were fixated on for the greatest amount of time were removed, performance, measured by the ability to take the 
most direct route to a goal location, significantly decreased. Thus, the landmarks that individuals fixated on seemed to be encoded into memory and useful for subsequent navigation. Fixation patterns also revealed that informative landmarks, like landmarks at decision points (i.e., intersections), were preferentially selected to look at over landmarks that provided less information. The authors concluded that eye fixations on landmarks during navigation are indicative of encoded information.

Despite these findings, it is unclear how fixation patterns in Andersen et al. (2012) and Hamid et al. (2010) would relate to fixation patterns in a larger-scale more realistic virtual world. Strayer, Drews, and Johnston (2003) used dynamic eye tracking in a highly realistic virtual environment where participants drove in a driving simulator to investigate the distracting effects of talking on a cell phone while driving. Eye tracking results revealed no significant differences in fixations to billboards on the side of the road between a group that drove while simultaneously conversing on a cell phone and a group that drove without a secondary task. However, recognition memory task performance that evaluated memory for the billboards showed that those who were talking on a cell phone remembered fewer billboards than controls that did not talk on a cell phone. Evidently, the distracted drivers had directly fixated on the signs that they did not remember, suggesting that the secondary task of talking on a cell phone did not interfere with eye movements but interfered with memory encoding and impaired memory performance later on. These results suggest that not everything to which visual attention is allocated is encoded in memory and contrast with those of Andersen et al. and Hamid et al., who demonstrated a link between eye fixations and memory. Therefore, the importance of 
looking at landmarks for forming an accurate cognitive map during navigation in a realistic virtual environment remains unclear.

In order to investigate the cognitive processes underlying individual differences in cognitive map accuracy, the current study investigated whether there are individual differences in where visual attention is allocated while navigating. Specifically of interest was whether individuals who form accurate cognitive maps of a novel virtual environment look at certain landmarks more than others in comparison with individuals who create less accurate cognitive maps. Participants completed a series of paper-andpencil and computer-based spatial tasks. First they rated their spatial abilities using the Santa Barabara Sense of Direction scale (SBSOD; Hegarty, Richardson, Montello, Lovelace, \& Subbiah, 2002), which has been shown to correlate with performance on large-scale navigation tasks (Hegarty et al., 2002; Kozhevnikov \& Hegarty, 2001). Next, they completed a small-scale test of spatial perspective-taking ability using the Spatial Orientation Test (SOT; Hegarty \& Waller, 2004), which has been associated with accuracy of cognitive map representations (Hegarty \& Waller, 2004; Sutton, Buset, \& Keller, 2014). After completing these tests, participants freely explored the virtual town, Silcton (Weisberg et al., 2014), and were instructed to pay special attention to 8 target buildings that were dispersed throughout the town. During exploration, participants' eye movements were recorded. Afterwards participants completed four tasks that assessed memory for Silcton: a landmark recognition task, a direction estimation task, a map building task, and a route construction task. The route construction task was included to provide another measure of cognitive map accuracy by requiring participants to create shortcuts between buildings (Bennett, 1996). 
Participants in the current study freely explored Silcton, meaning they could choose where to travel in the environment. This is in contrast to methods used in work by Ishikawa and Montello (2006), Weisberg et al. (2014), and Weisberg and Newcombe (2016). In these studies, participants were restricted to specific routes during the exploration process. Recent research by Sutton, Vollebregt, and Grogan (2016) indicates that performance on pointing and map-building tasks was significantly better in a free exploration paradigm relative to learning via specific routes. It is believed that free exploration allows an individual to experience more perspectives of objects in the environment and test hypotheses regarding the location of landmarks, facilitating the creation of a more accurate representation of the environment.

It was predicted that self-ratings of spatial ability would be associated with performance on tasks assessing memory for Silcton, and that perspective-taking ability would be associated with cognitive map accuracy. It was also predicted that performance on tasks that assessed memory for Silcton would be associated with each other. A number of participants were identified as being among participants with the most accurate cognitive maps or the least accurate cognitive maps. Those with the most accurate cognitive map representations were expected to also have better performance on the landmark recognition task and the route construction task than those who had inaccurate cognitive map representations. It was predicted that participants with highly accurate cognitive maps would direct more looking time to Silcton target buildings, non-target buildings, and objects. That is, individuals who formed the most accurate cognitive maps should look at all types of landmarks in the environment more than individuals who formed the least accurate cognitive maps. 


\section{Method \\ Participants}

Eighty-five participants (43 male and 42 female) were recruited for the study via posters displayed on the University of Western Ontario campus and a listing on the Department of Psychology research participation pool website. For ease of eye-tracking data collection and quality, only participants who had normal or corrected-to-normal vision with contact lenses were permitted to participate in the study. Due to technical errors or complications with some participants' contact lenses, data for five participants were not reliable (due to a periodic loss of eye tracking signal), thus the final sample consisted of data from 80 participants ( 40 male and 40 female; age $M=23.46$ years, $S D=$ 5.49). Some participants received $\$ 15$ in compensation for participating in the study and others received partial course credit. The study was approved by the University of Western Ontario Non-Medical Research Ethics Board.

\section{Materials and Procedure}

After providing written informed consent, participants completed a series of assessments, including scales, questionnaires, and tests. Assessments were ordered to ensure that completion of a particular assessment would not affect responses on subsequent assessments. With the exception of the paper-based tasks and Silcton exploration, all assessments were presented on a 19" desktop monitor connected to a laptop (Samsung R525, Samsung Electronics, Suwon, South Korea) running Windows 7 64-bit with an AMD Phenom II Quad-Core N970 2.2 GHz Processor, an AMD Radeon HD 6600M Graphics card (Advanced Micro Devices, Sunnyvale, CA), and an external mouse and keyboard. Silcton was presented on a 21" CRT monitor (1024 x 768 px, 
$1000 \mathrm{~Hz}$ sampling rate) which sat approximately 70 centimeters in front of the participant and was run on an ASUS computer operating Windows 10 64-bit with an AMD Athlon 64 X2 Dual Core 6000+ 3.15 GHz Processor, a Nvidia GeForce GTX 660 Graphics card, and a mouse and keyboard. Each experimental session lasted approximately 1.5 hours.

Demographic questionnaire. Participants completed an 8-item paper-based demographic questionnaire. On this questionnaire they provided information on age, gender, current year in university, whether they moved from a different city to attend university, and their modes of transportation (ride in car, drive car, take city bus, ride bicycle, and/or walk) including the most frequent mode of transportation. Additionally, they responded to questions regarding the navigation aids they used (car navigation system with map display, phone navigation system with map display, car navigation system with audio directions, phone navigation system with audio directions, paper map, I don't use navigation aids, ask someone for directions, and/or other), whether they had a drivers licence, how frequently they played video games, and types of video games played. Video game playing frequency was classified on a 5-point Likert scale ranging from zero (Less than once per week) to four (More than 6 times per week).

Santa Barbara Sense of Direction Scale. The next task was to complete the Santa Barbara Sense of Direction Scale (SBSOD; Hegarty, Richardson, Montello, Lovelace, \& Subbiah, 2002). Participants responded to the 15 -item assessment by rating their own spatial abilities on a 7-point Likert scale. The scale consisted of items such as "I am very good at reading maps," and "I very easily get lost in a new city."

Spatial Orientation Test. Next, the Spatial Orientation Test (SOT) (Hegarty \& Waller, 2004), a 12-item paper-and-pencil assessment, measured spatial perspective- 
taking ability using a static array of objects that was visible on the page for each item. For each item, participants were instructed to assume a heading direction within the object array (e.g., "Imagine you are standing at the car and facing the traffic light."). Then, they were instructed to indicate the direction of a third object in the array ("Point to the stop sign.”) by rotating their imagined orientation to match a given line on a circle and drawing a line from the centre of the circle to its perimeter. This drawn line indicated the direction of the third object. An error score was calculated for each item based on the average degrees between the correct bearing and the participants' response bearing for all items.

Direction estimation practice task. As practice for a later direction estimation task, participants completed a direction estimate practice task using buildings from the University of Western Ontario campus. Participants were shown a circle in Microsoft Powerpoint with an image of the building they were to imagine that they were standing near located in the centre of the circle and an image of the building that they were to imagine that they were facing located at the top of the circle. Then, they were instructed to place images of three other buildings on the perimeter of the circle in the appropriate direction with the given heading direction in mind. If a participant incorrectly completed the task or did not understand, further explanation and the correct answers were provided until they confirmed that they understood.

Silcton exploration task. Prior to Silcton (Weisberg et al., 2014) exploration, each participant's eye movements were calibrated. This was completed according to specifications for the EyeLink 1000 system with desktop mount via pop-up calibration mode (SR Research Ltd., Mississauga, Ontario, Canada). Participants completed an 
initial calibration trial by looking at dots on the screen, which moved to various locations. A second calibration trial was used to validate the results of the first. Immediately after calibration, continuous eye tracking and screen recording began in order to later produce a video of the participant's view while traveling in Silcton with eye tracking data overlaid. Eye movements were sampled continuously at a rate of $2000 \mathrm{~Hz}$ for the duration of exploration. Movement within Silcton was controlled using the up (forward) and down (backward) arrow keys on the keyboard, along with the mouse, both of which, when used simultaneously, guided the participant's horizontal and vertical viewpoints and direction of travel. Participants first practiced "walking" in Silcton to ensure they were comfortable with the controls. Participants were then given a minimum of 10 and a maximum of 20 minutes to freely explore the realistic virtual town. Free exploration mode in Silcton enables an individual to walk anywhere, including on the paths, off the paths, on the grass or parking lots, between buildings, and along the perimeter of the town. The majority of participants traversed most of these areas. Participants were instructed to remember the names and locations of eight buildings (Batty House, Lynch Station, Harris Hall, Tobler Museum, Sauer Center, Snow Church, Golledge Hall, and Harvey House) in Silcton, each of which was marked with a blue diamond that hovered over the path directly in front of the building. Figure 1 shows the layout of the 8 target buildings. A sign in front of each building showed the building name, and participants were also provided with a list of the 8 building names on a sheet of paper. For analysis, eye tracking and screen recording data were later trimmed to the beginning of each participants first "step" forward in Silcton, as some participants had questions between the end of calibration and prior to exploration. 


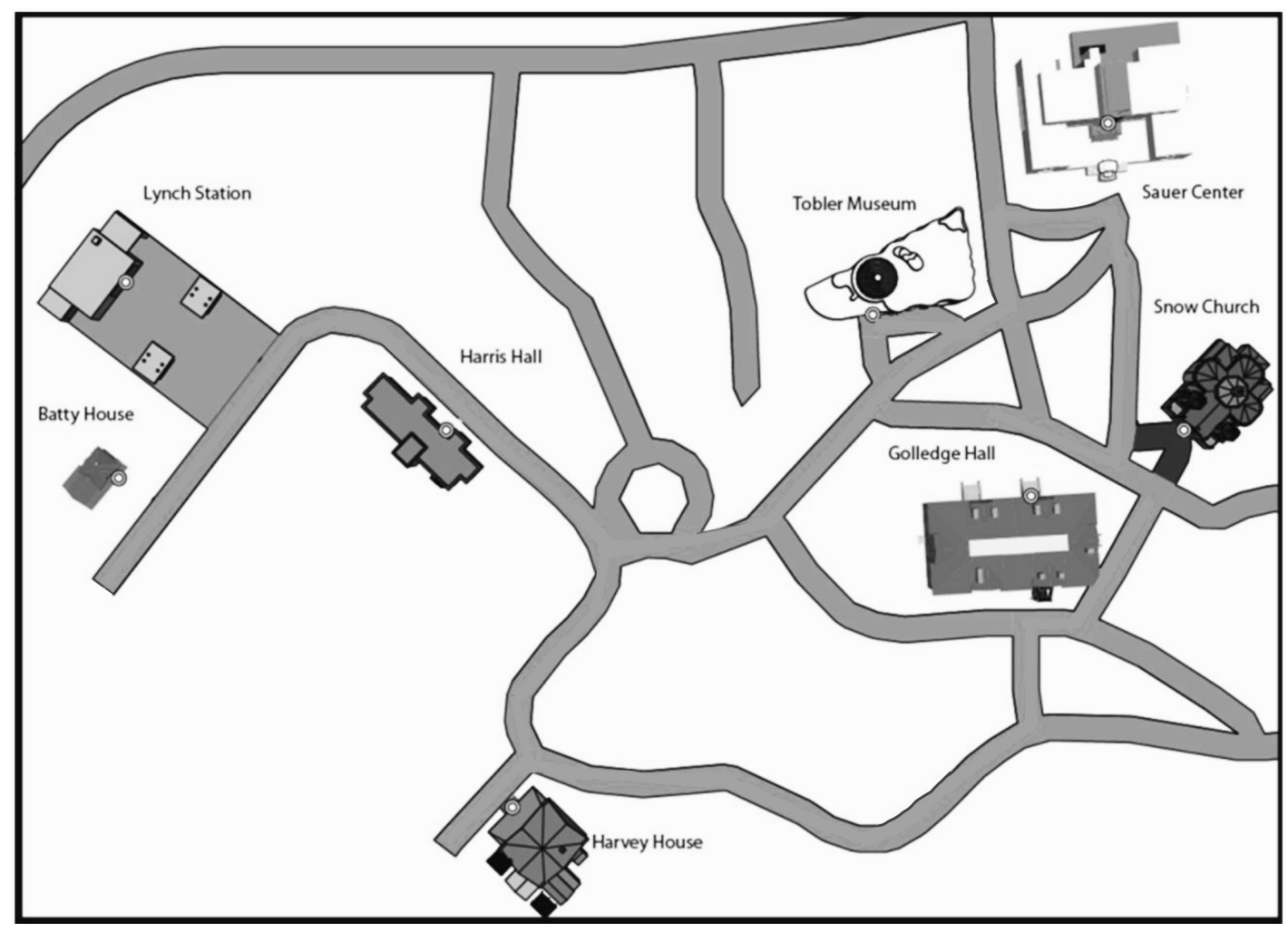

Figure 1: Silcton layout.

Locations of the 8 target buildings in Silcton. Note that Silcton consists of many more buildings and objects than the 8 target buildings shown here. Image adapted from Weisberg et al. (2014).

Silcton landmark recognition task. After the exploration period, participants completed a recognition task where randomized images of buildings and objects from Silcton and foils were presented in random order. In total, there were 32 trials: 11 buildings from Silcton, 5 objects from Silcton, and 16 foils. Foils consisted of items that would be typically found in a virtual town but were absent from Silcton (e.g., a mailbox, a fountain, other buildings that looked similar to those in Silcton). Each item remained on the screen until the participant made a response (indicating either yes this was a building/object in Silcton, or no this is a new building/object). There was no time limit to 
respond, but participants were instructed to press either key as quickly and as accurately as possible. The next item appeared immediately following a response. Stimuli were presented using E-Prime 2.0 software (Psychology Software Tools, Pittsburgh, PA). Data were analyzed as a discrimination index $\left(P_{\mathrm{r}}\right)$ calculated as the proportion of hits minus false alarms, as recommended by Snodgrass and Corwin (1988).

Direction estimation task. Next, participants completed a direction estimation task (Weisberg et al., 2014) using the 8 target buildings from Silcton. This task was similar to the direction estimation practice task. Participants were shown a circle on the screen with a heading direction (e.g., "Imagine you are standing at Batty diamond facing Lynch diamond.”). Then, participants were instructed to place the labels of the eight buildings around the perimeter of the circle in their appropriate directions. There were 8 trials, where each trial had a different pair of buildings with each building serving as the standing point once and the facing direction once.

Silcton map building task. Following completion of the direction estimation task, participants were presented with a map-building task (Weisberg et al., 2014). Participants were shown a blank two-dimensional box along with overhead bird's-eye images of the eight Silcton buildings. Participants were asked to place the buildings in their appropriate locations within the box. Accuracy was calculated automatically through the Silcton software via a bidimensional regression procedure (Friedman \& Kohler, 2003) which resulted in an $R^{2}$ value with a potential range from $0-1.0$. The procedure compares the similarity between the map completed by the participant and a map with the buildings in the correct locations. 
Silcton route construction task. In the final task, participants constructed 3 shortcut routes through Silcton. For each route, participants were given a starting location (e.g., Batty House) placed at the beginning of a horizontal line and an ending location (e.g., Sauer Center) placed at the end. Participants then dragged and dropped buildings onto the line to indicate which of the remaining six buildings they would encounter between the two buildings, and the order they would encounter them in, if they were to take the shortest route possible. Accuracy for both correct selection of buildings and order was scored using a point system. For each route, the number of points that participants received for each correct building was a proportion of the total number of correct buildings that completed that route. For example, if there were three buildings on the shortest possible route, participants received 0.33 points for each correct building so that the maximum number of points a participant could earn was 1.0. A fraction of a point (0.33) was deducted for each instance where extra buildings were included, buildings were missing, or if they were out of order, however the minimum possible score that could be obtained was 0 .

\section{Analysis of Eye Movement Data}

Eye tracking data for 32 participants were scored by the experimenter and a research assistant using DataViewer ${ }^{\mathrm{TM}}$ software (SR Research Ltd., Mississauga, Ontario, Canada). The primary experimenter scored eye tracking data for 25 participants, while data for 7 participants were scored by the research assistant. The research assistant was unaware of both the hypotheses of the study and the groups (participants with accurate cognitive maps, participants with inaccurate cognitive maps) to which participants belonged (details on the 32 participants selected for eye tracking scoring are below). 
Scoring practice was administered for the research assistant to ensure that scoring was as consistent as possible between scorers. Both scorers adhered to a scoring protocol. The scoring protocol for each participant involved replaying the recorded Silcton exploration file frame-by-frame and creating interest areas for each item that was fixated on. An interest area was a box that was drawn over an item (building/object) in Silcton when a participant looked at that item. The interest area was named according to the item and remained active for the duration that the participant looked at the item. When the participant diverted their gaze from the item, the active period for the interest area ended. This process was repeated for the entirety of the exploration video. In dynamic eye tracking, fixations from which visual information can be obtained and stored in memory are believed to range from 200 - 400 ms (Salvucci \& Goldberg, 2000), thus fixations under a $200 \mathrm{~ms}$ threshold were excluded from the analysis, consistent with other dynamic eye tracking work (Lahiri, Trewyn, Warren, \& Sarkar, 2011; Munn, Stefano, \& Pelz, 2008). Fixations to items in Silcton were grouped into one of four categories: Target buildings (any of the eight buildings that were marked with diamonds in Silcton), Nontarget buildings (buildings other than the target buildings in Silcton), Objects (items such as fire hydrants, cars, trees, and benches), and Other (the ground, sky, and items too far off in the distance that they could not be recognized).

\section{Statistical Analyses}

All data, except eye-tracking data, for the entire sample of 80 participants were analyzed using Pearson correlation analyses. Variables included age, sex, whether the participant moved to attend university, video game playing frequency, SBSOD score, SOT score, landmark recognition task score, direction estimation error score, map 
building accuracy score, and route construction task score. In addition, 32 participants were selected and classified as participants with the most and least accurate cognitive maps in the sample (see Participant Classification below for more detailed information). Two-tailed independent $t$ tests were performed to evaluate differences between the participants with accurate cognitive maps and the participants with inaccurate cognitive maps on composite cognitive map error scores, the SBSOD, the SOT, video game playing frequency, the landmark recognition task, the route construction task, and time spent navigating Silcton. Then, a 4 (Silcton item type: Target buildings, Non-target buildings, Objects, Other) x 2 (Group: Accurate cognitive maps, Inaccurate cognitive maps) mixed analysis of variance (ANOVA) was conducted to determine whether looking time to Silcton item categories varied by group.

\section{Results Full Sample}

Mode of travel reports. Twenty-nine percent of participants reported taking the city bus as their most frequently used mode of transportation. Walking was the next most frequent mode of transportation with $21 \%$ of participants, followed by driving a car at $16 \%$, and riding a bicycle and riding in a car both at $5 \%$. Five percent of participants did not report their most frequent mode of transportation.

Correlation analyses. Table 1 shows two-tailed Pearson correlation coefficients and significance values for age, sex, move for university, video game playing, SBSOD, SOT, the landmark recognition task, direction estimation task, map building task, and the route construction task scores. There was a significant negative correlation between how often video games were played $(M=1.44, S D=1.47)$ and age $(M=23.46, S D=5.49)$, 
where more frequent video game use was associated with being younger. There was also a significant negative correlation between video game frequency and sex, indicating that males played video games more often than females. As can be seen in Table 1, video game frequency was not significantly associated with any other measures, particularly measures for memory of Silcton, indicating that better performance on Silcton measures was not associated with playing video games.

A significant correlation between sex and landmark recognition task performance revealed that males $(M=.44, S D=0.20)$ recognized more landmarks from Silcton than females $(M=.35, S D=.20)$. Sex was also correlated with SBSOD ratings (overall $M=$ 4.80, $S D=1.05$ ) where higher SBSOD ratings were associated with being male (male: $M$ $=5.09, S D=0.97$; female: $M=4.51, S D=1.06$ ). Self-reported ratings on the SBSOD for all participants were significantly associated with error on the direction estimation task $(M=71.94, S D=9.81)$ indicating that as SBSOD ratings increased, error on the direction estimation task decreased (i.e., accuracy increased).

Error on the Spatial Orientation Test $(M=26.02, S D=18.81)$ was significantly associated with all measures for memory of Silcton. In particular, SOT performance was negatively associated with landmark recognition $(M=.39, S D=.21)$, map building ( $M$ $=.62, S D=.26)$, and route construction $(M=.45, S D=.35)$, meaning higher accuracy on the SOT was associated with recognizing more landmarks, building more accurate maps of Silcton, and constructing more accurate routes of Silcton, respectively. There was also a significant correlation between SOT performance and direction estimation performance $(M=71.94, S D=9.81)$ such that better accuracy on the SOT was associated with better accuracy in estimating the directions between landmarks in Silcton. 


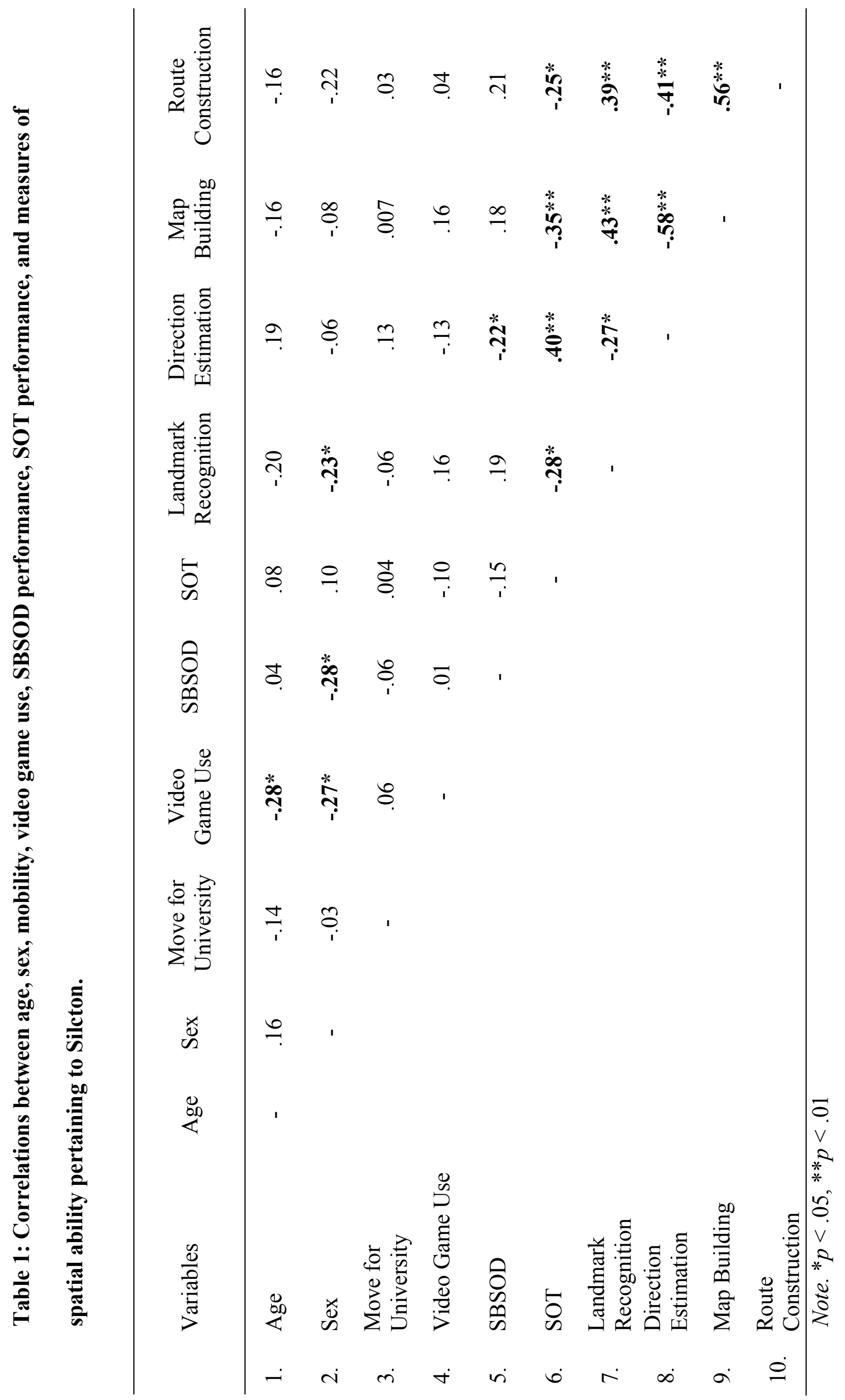




\section{Participant Classification}

Participants were classified according to their performance on the Silcton assessments that specifically tested cognitive map accuracy (i.e., the direction estimation task and the map building task). A composite cognitive map error measure was calculated for each participant. This measure was based on the error measure obtained from performance on the direction estimation task, as well as the map-building accuracy score, which was converted to an error score via reverse scoring and conversion to a whole number. The two error values were then summed to calculate a single error measure of cognitive map accuracy. For example, a map-building score of .957586 was reverse scored and converted to a whole number of 4.241408 by multiplying .957586 and 100 and subtracting it from 100. Then, 4.241408 was added to the participant's direction estimation score of 54.553564 which resulted in a rounded composite cognitive map error score of 58.79. Composite map error scores ranged from 58.79 to 185.23 (see Figure 2), with higher scores indicating a less accurate cognitive map. Participants in the top $20 \%$ (most accurate) and bottom 20\% (least accurate) on the composite cognitive map error score were identified, resulting in 16 participants in the accurate cognitive map group and 16 in the inaccurate cognitive map group. Eye tracking data were only examined for these individuals with the most and least accurate cognitive maps, $\mathrm{N}=32$. 


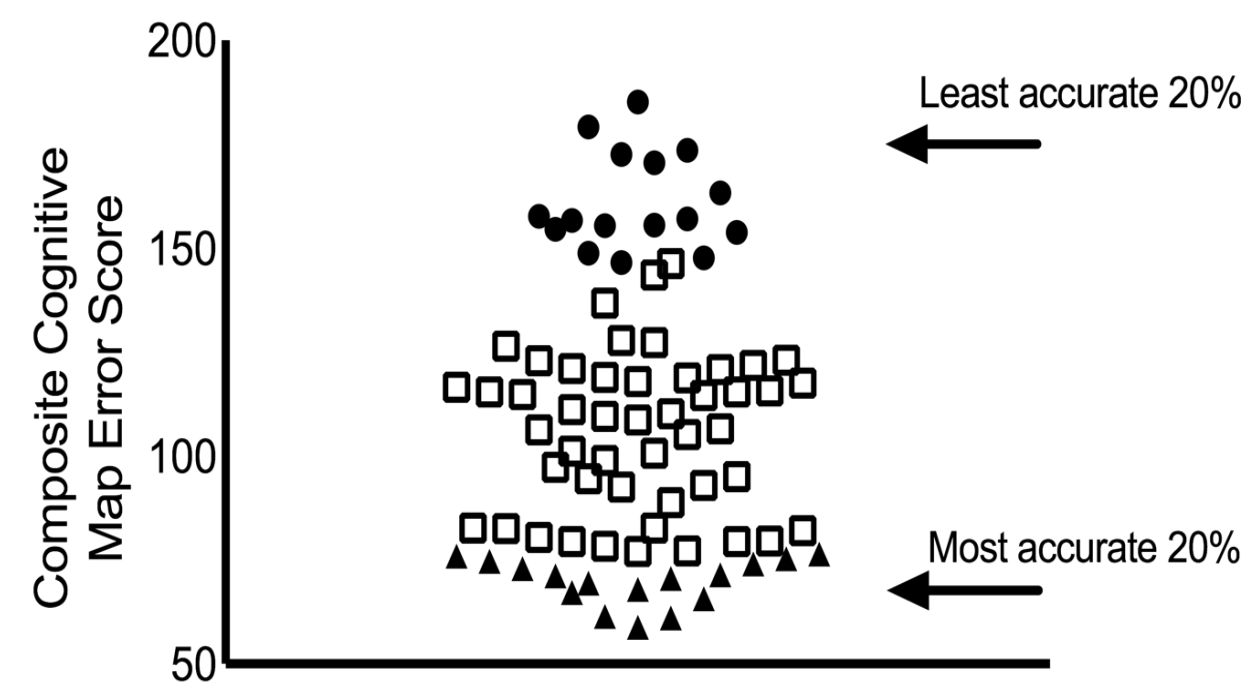

Figure 2: Participant classification.

Selection and classification of participants into most and least accurate cognitive map groups based on calculated cognitive map error score using performance on the direction estimation and map building tasks. Points (circles, triangles, and squares) represent individual composite cognitive map scores. Triangles represent the top $20 \%$ of participants with the most accurate cognitive maps. Circles represent the bottom $20 \%$ of participants with the least accurate cognitive maps. Squares represent the middle $60 \%$ of participants.

\section{Comparisons of Individuals with Accurate and Inaccurate}

\section{Cognitive Maps}

Cognitive assessments. As expected, a Levene's corrected, $F(1,30)=9.65, p$ $=.004, t$ test revealed that Silcton composite cognitive map scores were significantly more accurate for individuals with accurate cognitive maps $(M=69.63, S D=5.57)$ than individuals with inaccurate cognitive maps $(M=161.14, S D=11.66)$ navigators, $t(21.50)$ 
$=-28.32, p<.001, d=-54.85$. The means, standard errors, and individual scores for those with accurate and inaccurate cognitive maps are shown in Figure 3.

Reports of the most frequent mode of transportation showed a similar pattern for both individuals with accurate cognitive maps and individuals with inaccurate cognitive maps. Both accurate and inaccurate cognitive map groups reported taking the city bus as their most frequent mode of transportation (accurate: $37.5 \%$, inaccurate: $50 \%$ ), followed by walking (accurate: $31.25 \%$, inaccurate: $18.75 \%$ ), then driving a car (accurate: 18.75 , inaccurate: $12.5 \%$ ), riding a bicycle (accurate: $12.5 \%$, inaccurate: $6.25 \%$ ), and riding in a car (accurate: $0 \%$, inaccurate: 6.25\%). A Fischer's exact Chi-square test revealed that most frequent mode of transportation reported was not significantly associated with group, $p=.77$.

Independent two-tailed $t$ tests were conducted to compare individuals with accurate cognitive maps to individuals with inaccurate cognitive maps on video game frequency, SBSOD ratings, and performance on the SOT and route construction task (see Figure 4). As can be seen in Figure 4d, route construction accuracy was significantly better for individuals with accurate cognitive maps $(M=.81, S D=.22)$ compared to individuals with inaccurate cognitive maps $(M=.20, S D=.31), t(30)=6.46, p<.001, d$ $=12.51$, indicating that individuals with accurate cognitive maps were better able to create shortcuts through Silcton than individuals with inaccurate cognitive maps. Shown in Figure 4a, those with inaccurate cognitive maps rated their spatial abilities on the SBSOD $(M=4.53, S D=1.24)$ numerically lower but not significantly different than those with accurate cognitive maps $(M=5.12, S D=.77), t(30)=1.63, p=.11, d=3.16$. 


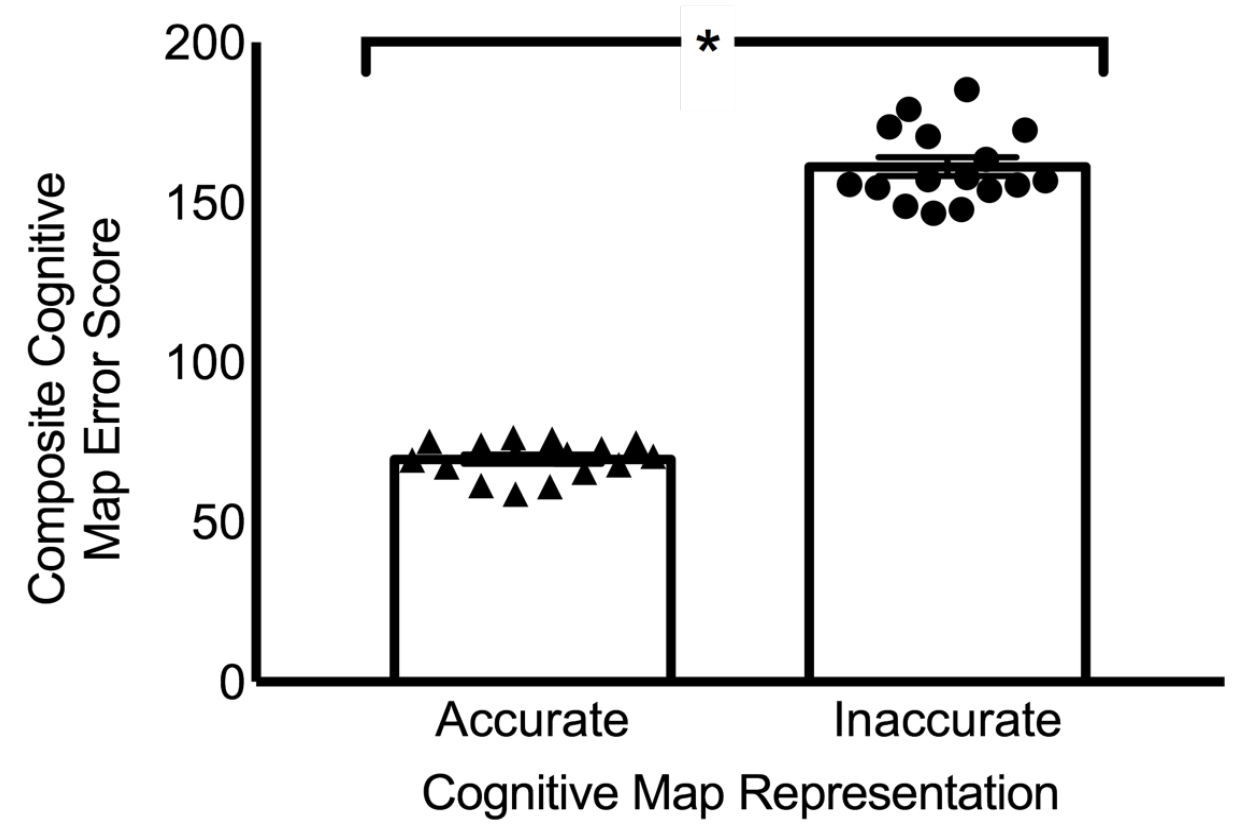

\section{Figure 3: Composite cognitive map error scores.}

Composite cognitive map error scores for participants selected from the original full sample as falling in the most accurate $20 \%(\mathrm{~N}=16)$ and least accurate $20 \%$ of cognitive maps in the sample $(\mathrm{N}=16)$. Triangles represent individuals with accurate cognitive maps. Circles represent individuals with inaccurate cognitive maps. Error bars represent standard error of the mean.

For the SOT (see Figure 4b), an independent $t$ test not assuming homogenous variances, Levene's $F(1,30)=13.51, p=.001$, was calculated and indicated that SOT performance was significantly better (i.e., lower error) for participants with accurate cognitive maps $(M=16.74, S D=8.26)$ than participants with inaccurate cognitive maps $(M=35.33, S D$ $=21.45), t(19.36)=-3.24, p<.01, d=-6.27$, indicating better small-scale perspectivetaking ability for the group with accurate cognitive map representations. Finally, video game playing frequency (Figure 4c) was not significantly different between those with 
A

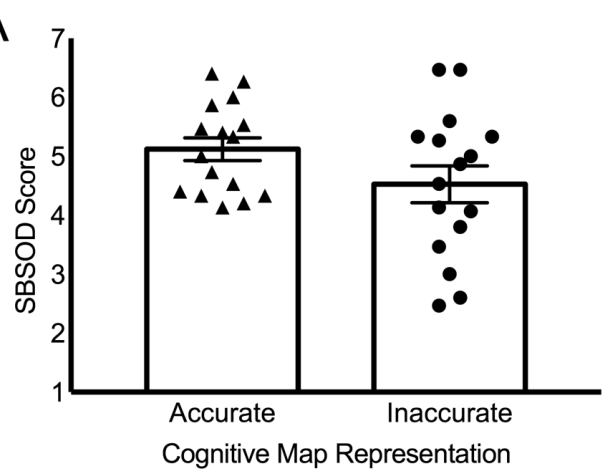

C

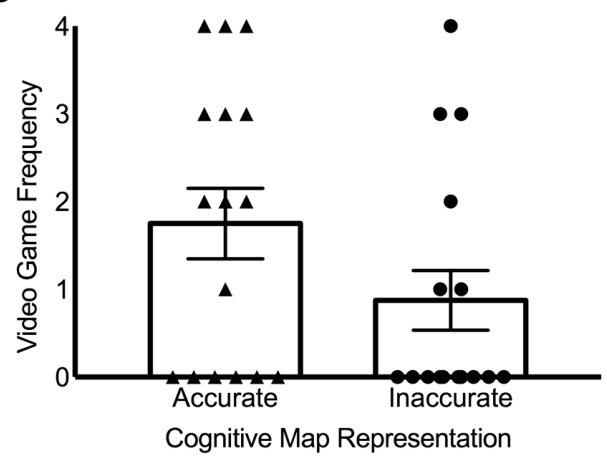

B
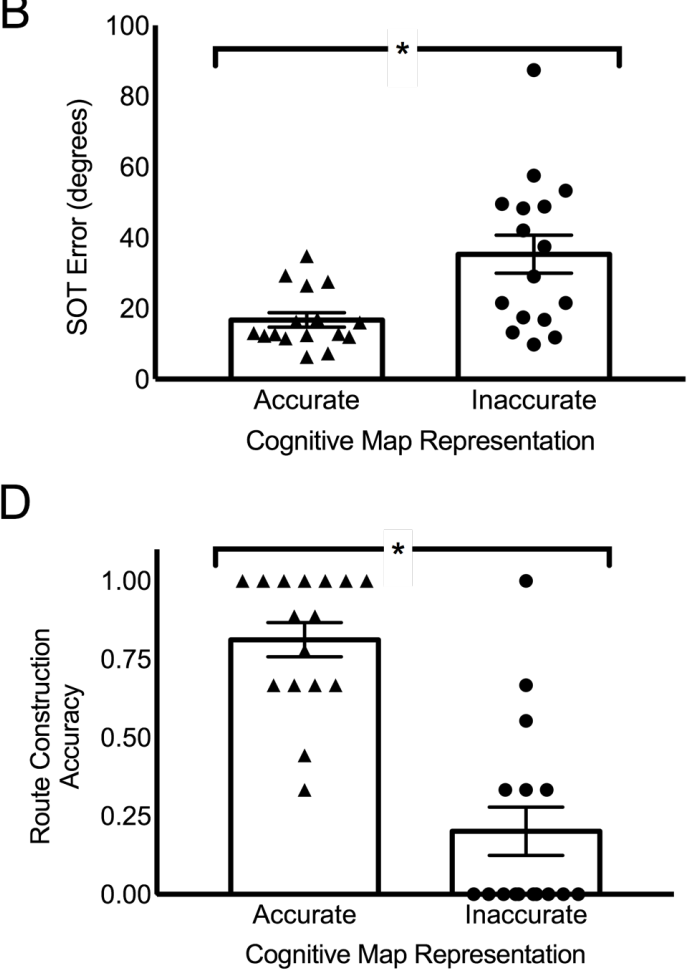

Figure 4: Performance on assessments of spatial ability and gaming frequency.

(A) Self-ratings on the SBSOD scale for participants with accurate cognitive map representations and participants with inaccurate cognitive map representations. (B) SOT performance for participants in the accurate and inaccurate cognitive map groups. (C) Video game playing frequency for participants with accurate cognitive map representations and participants with inaccurate cognitive map representations. (D) Performance on the route construction task for participants in the accurate and inaccurate cognitive map groups. Triangles represent individuals with accurate cognitive map representations. Circles represent individuals with inaccurate cognitive map representations. Error bars represent standard error of the mean. 
accurate cognitive maps $(M=1.75, S D=1.61)$ and those with inaccurate cognitive maps $(M=.88, S D=1.36), t(30)=1.66, p=.11, d=3.21$.

Eye tracking data. Each participant's looking time to each Silcton item category was converted to a proportion based on total looking time for that participant. Participants with accurate cognitive map representations differed from participants with inaccurate cognitive map representations on looking times to items in Silcton in some respects but not others. A mixed 2 (Group: accurate cognitive map, inaccurate cognitive map) x 4 (Silcton item type: target buildings, non-target buildings, objects, other) ANOVA with Group as the between subjects factor and Silcton items as the repeated factor was conducted on the proportion of total looking time data. The Greenhouse-Geisser correction for $F$ was used for all repeated measures effects. The ANOVA revealed a significant main effect of Silcton items, $F(1.29,38.69)=288.54, p<.001, \eta_{\mathrm{p}}{ }^{2}=.91$. Bonferroni-corrected post hoc tests showed that the proportion of time spent looking at target buildings $(M=.09, S D=.04)$ was not significantly different than the proportion of time spent looking at non-target buildings $(M=.08, S D=.03), p=.18$. The proportion of looking time to target buildings was significantly less than looking time to objects $(M$ $=.26, S D=.07), p<.001$, and less than items in the other category $(M=.57, S D=.11)$, $p<.001$. The proportion of time spent looking at non-target buildings was also significantly less than time spent looking at objects, $p<.001$, and items in the other category, $p<.001$. Looking time for objects was significantly less than time spent looking at items in the other category, $p<.001$. All other comparisons were not statistically significant, $p \mathrm{~s}>.05$. The main effect of group was not significant, as looking times were converted to proportions and the mean looking time for each group was 1.0. 
The Group x Silcton Items ANOVA on looking time data also revealed an interaction, where the proportion of time spent looking at different categories of items in Silcton was dependent on the whether an individual had an accurate or inaccurate cognitive map representation, $F(1.29,38.69)=4.09, p=.04, \eta_{\mathrm{p}}{ }^{2}=.12$. Figure 5 depicts the Group x Silcton Items interaction.

A simple main effects analysis for the effect of group at each Silcton item category was conducted to investigate which items showed differences in proportion of looking time between those with accurate cognitive maps and those with inaccurate cognitive maps. For target buildings, looking times to target buildings by participants with accurate cognitive maps $(M=.10, S D=.04)$ and participants with inaccurate cognitive maps $(M=.08, S D=.04)$ did not differ significantly, $F(1,30)=2.05, p=.16$, $\eta_{\mathrm{p}}{ }^{2}=.00$. Looking times to non-target buildings were significantly different, indicating that those with accurate cognitive maps $(M=.10, S D=.03)$ spent significantly more time looking at non-target buildings than those with inaccurate cognitive maps $(M=.07, S D$ $=.02), F(1,30)=8.34, p=.007, \eta_{\mathrm{p}}{ }^{2}=.25$. Similar to the pattern for target buildings, looking time to objects in Silcton did not differ significantly between the accurate cognitive map group $(M=.27, S D=.07)$ and the inaccurate cognitive map group ( $M$ $=.24, S D=.06), F(1,30)=1.94, p=.17, \eta_{\mathrm{p}}^{2}=.07$. For Silcton items in the "other" category, the inaccurate cognitive map group $(M=.61, S D=.09)$ looked significantly longer at items in this category, such as the ground and the sky, than the accurate cognitive map group $(M=.53, S D=.11), F(1,30)=4.86, p=.04, \eta_{\mathrm{p}}{ }^{2}=.13$.

Next, to investigate whether the pattern of looking times to Silcton items varied within each group, a simple main effects analysis of proportion of total looking time to 


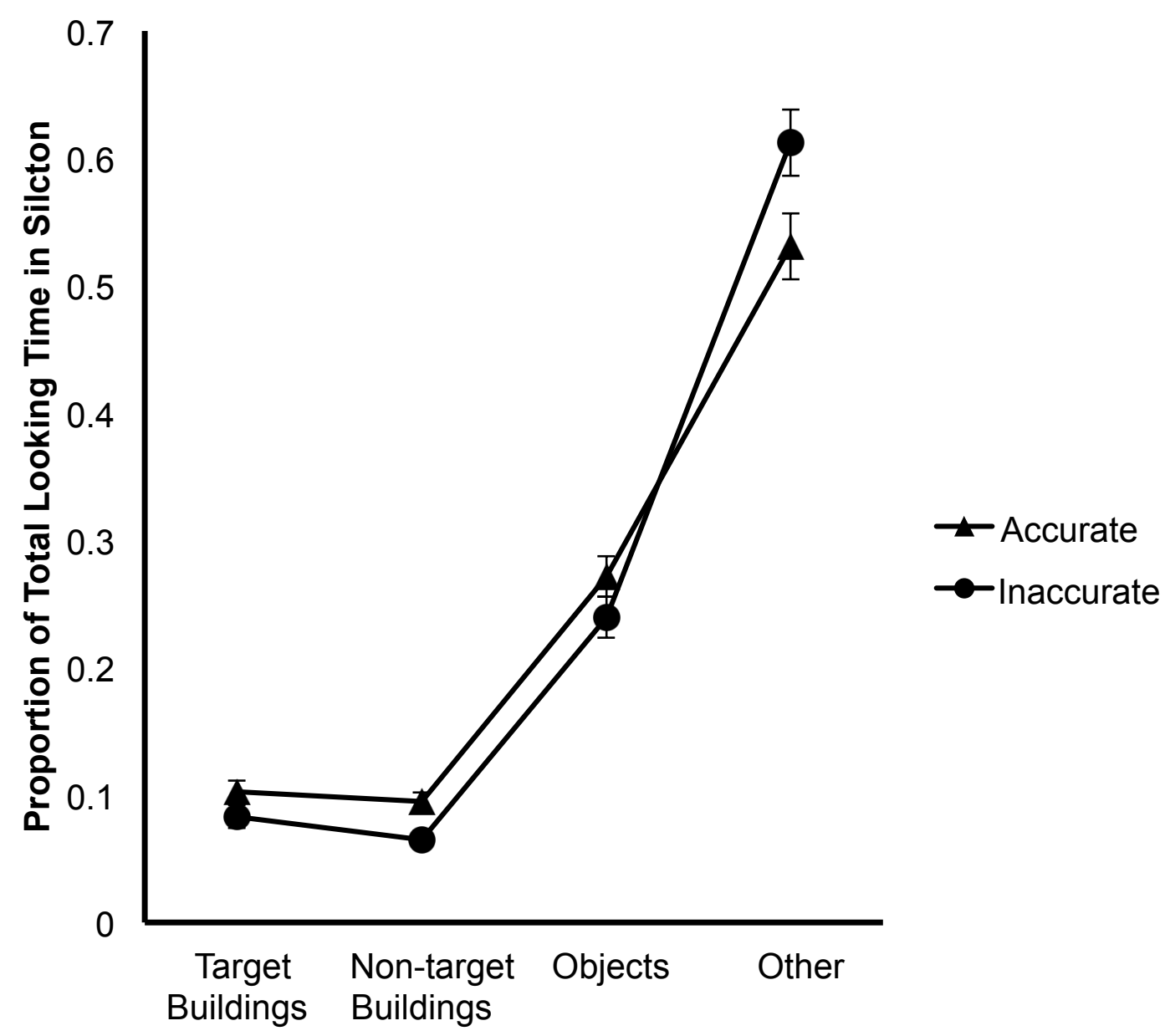
Silcton Item Type

\section{Figure 5: Attention to Silcton item categories.}

Proportion of looking time attributed to each of the four Silcton item categories for the accurate and inaccurate cognitive map groups. Triangles represent the accurate cognitive map group. Circles represent the inaccurate cognitive map group. Error bars represent standard error of the mean.

different Silcton items for each group was conducted. Proportion of total looking time to the Silcton item categories differed significantly for those with an accurate cognitive map, $F(3,90)=115.04, p<.001, \eta_{\mathrm{p}}{ }^{2}=.79$, and those with an inaccurate cognitive map, $F(3$, 
$90)=177.59, p<.001, \eta_{\mathrm{p}}{ }^{2}=.86$. Bonferroni-corrected post hoc analyses on the simple main effect for the group with accurate cognitive maps revealed that they spent more time looking at objects $(M=.27, S D=.07)$ than target buildings $(M=.10, S D=.04), p<.001$. They also spent significantly more time looking at items in the other category $(M=.53$, $S D=.11)$ compared to target buildings, $p<.001$ and more time looking at items in the other category than non-target buildings $(M=.09, S D=.03), p<.001$. Participants with an accurate cognitive map also spent significantly more time looking at items in the other category than looking at objects, $p<.001$, but more time looking at objects than nontarget buildings, $p<.001$. There was no significant difference between time spent looking at target buildings compared to non-target buildings, however, $p=1.00$.

Like the group with accurate cognitive maps, the group with inaccurate cognitive maps also spent the majority of their time looking at items in the other category $(M=.61$, $S D=.09)$. The other category had a significantly higher proportion of looking time compared to target buildings $(M=.08, S D=.04), p<.001$, non-target buildings $(M=.06$, $S D=.02), p<.001$, and objects $(M=.24, S D=.06), p<.001$. Similar to the pattern for the group with accurate cognitive maps, the group with inaccurate cognitive maps spent less time looking at target buildings compared to objects, $p<.001$, and non-target buildings were also looked at significantly less than objects, $p<.001$. Similar to participants with an accurate cognitive map, time spent looking at target buildings did not significantly differ from time spent looking at non-target buildings, $p=.28$.

An analysis of performance on the landmark recognition task, via a two-tailed independent $t$ test, revealed that individuals with an accurate cognitive map $(M=.53, S D$ $=.17$ ) correctly distinguished significantly more Silcton items (buildings and objects) 
from foils than individuals with an inaccurate cognitive map $(M=.27, S D=.18), t(30)=$ $4.08, p<.001, d=7.91$. Figure 6 shows that although there were a few participants in the accurate cognitive map group that performed poorly on the recognition task, overall, individuals with an accurate cognitive map outperformed individuals with an inaccurate cognitive map.

Finally, it is possible that individuals with an accurate cognitive map simply spent more time in Silcton than individuals with an inaccurate cognitive map, since participants determined the length of the exploration period themselves (within the 10 - 20 minute allowance). Given a violation of Levene's test for homogeneity of variances, $F(1,30)=$ $4.88, p=.04$, an independent $t$ test not assuming homogenous variances was calculated and showed that exploration time for the accurate cognitive map group $(M=15.84 \mathrm{~min}$, $S D=2.99 \mathrm{~min})$ did not significantly differ from the inaccurate cognitive map group $(M=$ $16.13 \mathrm{~min}, S D=4.66 \mathrm{~min}), t(25.57)=-0.21, p=.84, d=-0.40$. 


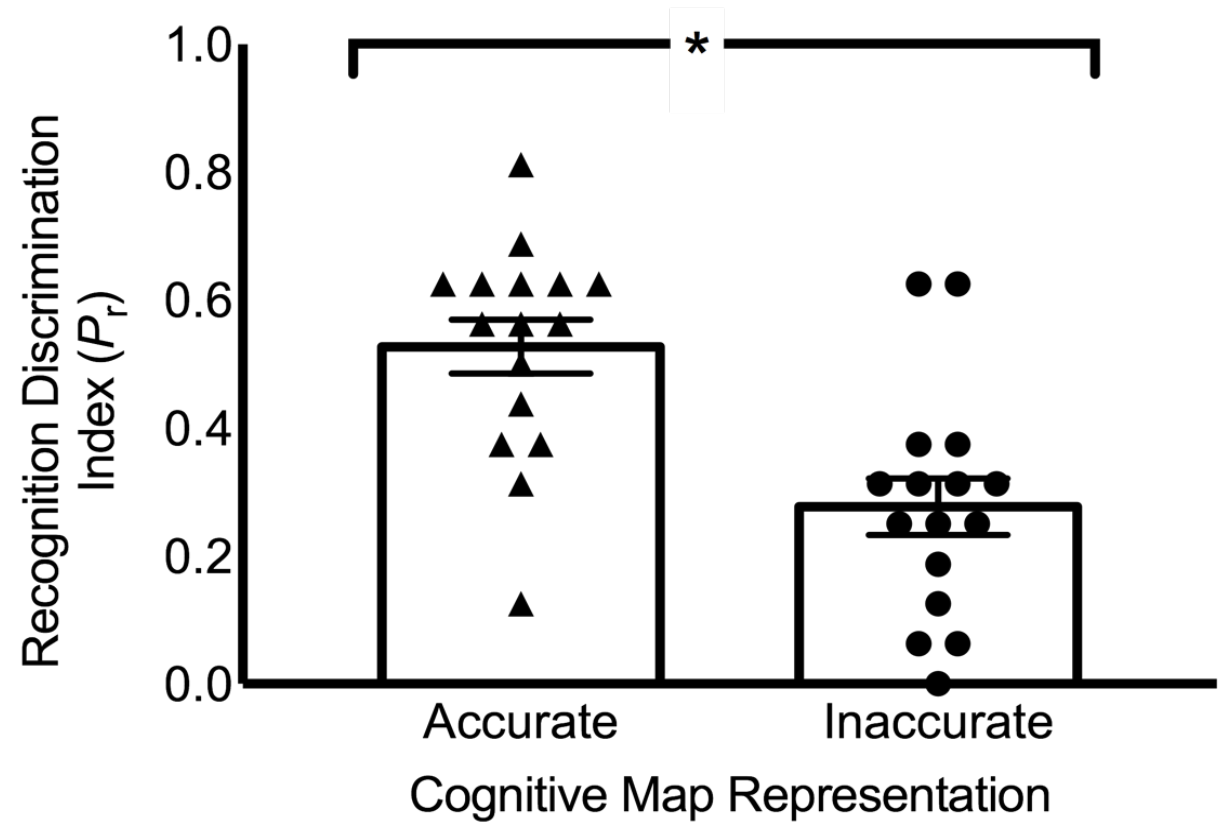

Figure 6: Landmark recognition task performance.

Triangles represent individuals with an accurate cognitive map. Circles represent individuals with an inaccurate cognitive map. Error bars represent standard error of the mean.

\section{Discussion}

The current study used dynamic eye tracking technology to measure fixations to landmarks within a novel virtual environment during a free exploration navigation task. The aim was to determine whether the ability to form an accurate mental representation of a novel environment is related to differences in the allocation of visual attention. Analyses of data from all 80 participants revealed sex differences where being younger and being male was associated with higher video game playing frequency. There was also a sex difference for the landmark recognition task and the SBSOD, where males performed better on the landmark recognition task and had higher self-ratings on the 
SBSOD. For the SBSOD, higher self-ratings were also associated with a more accurate ability to estimate the directions between buildings in Silcton. In addition, performance on the small-scale spatial perspective-taking SOT was related to performance on all tasks that assessed memory for Silcton.

After classifying selected participants into two groups based on cognitive map accuracy, analyses of performance on spatial assessments revealed that individuals with an accurate cognitive map had better composite cognitive map error scores, were more accurate in constructing shortcuts, and had better perspective-taking ability, yet both groups had similar self-ratings of spatial abilities and levels of video game playing frequency. During Silcton exploration, individuals with accurate and inaccurate cognitive maps looked at to-be-remembered target buildings for similar amounts of time, yet those with inaccurate cognitive maps correctly identified fewer items overall from Silcton than individuals with accurate cognitive maps. This pattern of similar looking times was repeated for objects, however, the group with accurate cognitive maps looked at nontarget buildings more than the group with inaccurate cognitive maps, and those with inaccurate cognitive maps looked at the sky and ground more than those with accurate cognitive maps. Both groups spent the same amount of time exploring Silcton.

In our samples of individuals with accurate and inaccurate cognitive maps, looking times to target buildings and objects in Silcton were similar in both groups, but different for non-target buildings and the other category. Interestingly, the groups differed in both spatial and non-spatial memory for items in all categories, whereby individuals with an accurate cognitive map were more accurate in distinguishing landmarks that were and were not in Silcton, constructing shortcuts through Silcton, and 
also had better small-scale perspective-taking abilities. Thus, the current findings resemble those of Strayer et al. (2003), where some individuals demonstrated a significant impairment in remembering items that they had directly looked at. Strayer et al. suggest that despite looking at the billboards while driving, the attention of drivers who were talking on a cell phone was diverted to the concurrent phone conversation, which produced memory interference. The fact that the current results show that landmarks in the environment were not remembered despite having looked directly at them, albeit without a secondary task, is a novel finding in the spatial cognition literature. They suggest that differences in cognitive map accuracy may not be due to differences in the landmarks that an individual looks at, but rather due to differences in general memory encoding and/or retrieval ability. Differences such as these further downstream from attention in information processing could affect cognitive map accuracy because an inability to remember the locations and visual features of landmarks in the environment would make it difficult to form a cognitive map of the environment since a cognitive map is based on landmark information.

Navigation is known to involve working memory processes (Wolbers \& Hegarty, 2010), as spatial and non-spatial landmark information needs to be updated when more information about the environment is acquired. In the current study, information may have passed through the attention stage, but was disrupted for individuals with inaccurate cognitive maps at the working memory encoding and/or retrieval stage. These findings add to mixed literature regarding the role of memory for navigation. Weisberg and Newcombe (2016) showed that weak navigators had poor working memory (both spatial and verbal) abilities, while Sutton, Keller, and Vollebregt (2017) showed a weak 
association between cognitive map accuracy and spatial working memory ability in a sample of teenagers. Therefore, working memory ability may be a contributing factor in cognitive map accuracy.

It is possible that individuals with inaccurate cognitive maps experienced information overload during the information encoding and/or retrieval stages. Navigation is a complex task, requiring an individual to pay attention to a variety of aspects both of one's self and items within the environment. An individual needs to maintain a sense of their location and update the locations of landmarks around them (Wolbers \& Hegarty, 2010), information that must be stored in memory and can later be used to make inferences when solving navigation tasks. The findings of the current study did not support Hamid et al.'s (2010) conclusion that individuals encode the landmarks they look at into memory, at least for individuals with inaccurate cognitive maps. This may be because individuals with inaccurate cognitive maps remember landmarks in different, perhaps less effective, ways. Previous research has indicated that in comparison with individuals who perform poorly on navigation tasks, those who perform well on navigation tasks do in fact use working memory in different ways to encode information about the environment (Ploran, Rovira, Thompson, \& Parasuraman, 2015; Wen, Ishikawa, $\&$ Sato, 2011; 2013). For instance, in a study where people learned routes from a video and completed memory interference tasks, good navigators used a combination of verbal and spatial working memory to store cognitive map knowledge, while poor navigators relied only on verbal working memory (Wen et al., 2011; 2013). In addition, Ploran et al. (2015) showed that success in finding targets in a large-scale navigation task was dependent on the recruitment of spatial working memory. Since memory encoding and/or 
retrieval ability might be responsible for differences in cognitive map accuracy, future work should further evaluate the relationship between working memory capacity and performance differences between those with accurate cognitive maps and those with inaccurate cognitive maps.

In addition to memory capabilities, good perspective-taking ability may play a critical role in forming an accurate cognitive map. There was a significant association between SOT performance and performance on all of the Silcton spatial measures in the current study. The relationship was evident for the landmark recognition task, the direction estimation task, the map building task, and the route construction task, in both the overall sample and after participants were classified as having an accurate or inaccurate cognitive map. Similar findings have been shown in our other work with a different virtual environment (Sutton et al., 2014). It is possible that good perspectivetaking ability allows an individual to form a more accurate cognitive map because one can imagine the location of landmarks in the environment from multiple viewpoints. In theory, this ability could potentially make scaling and interrelating various segments of the environment, a process proposed by Siegel and White (1975), easier, resulting in a more accurate map-like representation. Since a cognitive map is orientation-independent, visualization of the environment with good perspective-taking skills may facilitate localizing oneself in the environment, the direction one must travel to get to a goal destination, and visualizing the landmarks that one will encounter along the way through the recruitment of both the egocentric and allocentric reference frames. Good perspective-taking ability and use of both reference frames would result in superior navigation performance overall. 
The way individuals with inaccurate cognitive maps distributed their looking time in Silcton may have negatively affected their performance. Since individuals with inaccurate cognitive maps attributed less attention to non-target buildings, they may have consequentially received less contextual information about the environment. For instance, non-target buildings may assist in remembering the locations of the target buildings and aid an individual in building an accurate cognitive map. An individual may remember the location of the target building Sauer Center by remembering that it is next to the bank (a non-target building), which is also in close proximity to another target building known as Snow Church. The amount of time that individuals with inaccurate cognitive maps did not spend looking at non-target buildings seemed to be directed to items in the other category instead. Time spent looking at items in the other category, specifically the ground and the sky, might be considered time wasted, as these items should provide little information in terms of cues for navigation because they lack distinguishable features that would allow an individual to maintain a sense of their position in the environment. Therefore, directing less looking time to potentially informative landmarks and more looking time to uninformative features of the environment seems to significantly impair cognitive map accuracy.

Interestingly, self-ratings on the Santa Barbara Sense of Direction scale were not reflective of differences in cognitive map accuracy. Although, previous work has found that SBSOD ratings are associated with performance on large-scale navigation tasks requiring an accurate cognitive map (Hegarty et al., 2002; Kozhevnikov \& Hegarty, 2001), no such association was found in the current study. While there was a relationship between SBSOD ratings and performance on the direction estimation task, this 
relationship was not found in individuals with the most and least accurate cognitive maps. There was considerable variation in self-reported ratings of spatial abilities in the group with inaccurate cognitive maps. However, overall, despite their poor performance on the spatial tasks, those with an inaccurate cognitive map still rated their spatial skills on the SBSOD to be nearly as high as those with a highly accurate cognitive map. This finding may highlight a lack of awareness that individuals with inaccurate cognitive maps have of their own spatial abilities to the point that some individuals with inaccurate cognitive maps may have an inflated sense of their navigation abilities. In comparison with previous work with early career civil aviation pilots, a population with demonstrably more advanced spatial abilities than the average population (Sutton et al., 2014), both individuals with accurate and inaccurate cognitive maps in the current study rated their spatial abilities nearly as high as pilots. Thus, some individuals with inaccurate cognitive maps in our sample seem to have been overly confident in their perceptions of their own spatial abilities. Alternatively, participants' self-ratings might have been reflective of their true abilities but the tasks used in the current study may not have accurately probed real world performance. More research would be needed to rule out this possibility.

For assessing navigation abilities, virtual reality is a methodology with high validity in a laboratory setting and offers significant control over what is presented to the participant (Waller \& Greenauer, 2007). Unlike most previous research that has used simple virtual environments with dynamic eye tracking, the current study utilized a naturalistic virtual environment. However, despite Silcton's realistic features, the environment lacked moving objects (such as cars, birds, and people) that would normally attract attention in a real setting. In laboratory tasks assessing attention, stimuli with 
sudden onsets capture attention automatically (Yantis, 1993; Yantis \& Jonides, 1990). An individuals' spatial and non-spatial memory for landmarks would likely be more adversely affected if, while navigating a new city and paying special attention to landmarks attention is interrupted by a car that nearly hits the individual. To be certain, however, more research using dynamic eye tracking and manipulating the amount of moving stimuli in either virtual or real environments to examine the effect on spatial and non-spatial memory is needed.

In conclusion, cognitive map accuracy appears not to be associated with the landmarks that are looked at while exploring a new environment, but is, in part, associated with the ability to remember elements of the landmarks that one directs visual attention to and perspective-taking ability. The results indicated that some individuals experience significant memory difficulties for the landmarks that they look at. While the current study advances the field by providing evidence against the hypothesis that differences in cognitive map accuracy have their roots in differences in attention to landmarks, more research is required to tease apart potential differences in memory capabilities by exploring differences that may lie further downstream in information processing, such as whether information about landmarks is properly encoded or whether those with inaccurate cognitive maps are able to encode landmark information but experience trouble retrieving the information. This will further enhance our understanding of the factors that contribute to the ability to build an accurate cognitive map, conceivably leading to the development of interventions to improve navigation ability for those who struggle. 


\section{References}

Aguirre, G. K., \& D’Esposito, M. (1999). Topographical disorientation: A synthesis and taxonomy. Brain: A Journal of Neurology, 122, 1613-1628.

Allen, G. L., Kirasic, K. C., Siegel, A. W., and Herman, J. F. (1979). Developmental issues in cognitive mapping: The selection and utilization of environmental landmarks. Child Development, 50, 1062-1070.

Andersen, N. E., Dahmani, L., Konishi, K., \& Bohbot, V. D. (2012). Eye tracking, strategies, and sex differences in virtual navigation. Neurobiology of Learning and Memory, 97, 81-89.

Bennett, A. T. (1996). Do animals have cognitive maps? Journal of Experimental Biology, 199, 219-224.

Cohen, R., \& Schuepfer, T. (1980). The representation of landmarks and routes. Child Development, 51, 1065-1071.

Cousins, J. H., Siegel, A. W., \& Maxwell, S. E. (1983). Way finding and cognitive mapping in large-scale environments: A test of a developmental model. Journal of Experimental Child Psychology, 35, 1-20.

Curtis, L. E., Siegel, A. W., \& Furlong, N. E. (1981). Developmental differences in cognitive mapping: Configurational knowledge of familiar large-scale environments. Journal of Experimental Child Psychology, 31, 456-469.

Evans, G. W., Marrero, D. G., \& Butler, P. A. (1981). Environmental learning and cognitive mapping. Environment and Behaviour, 13, 83-104.

Foo, P., Warren, W. H., Duchon, A., \& Tarr, M. J. (2005). Do humans integrate routes into a cognitive map? Map- versus landmark-based navigations of novel shortcuts. Journal of Experimental Psychology: Learning, Memory, and Cognition, 31, $195-$ 215.

Friedman, A., \& Kohler, B. (2003). Bidimensional regression: Assessing the configural similarity and accuracy of cognitive maps and other two-dimensional data sets. Psychological Methods, 8, 468-491.

Gallistel, C. R. (1990). The organization of learning. Cambridge, MA: MIT Press.

Golledge, R. G. (1999). Wayfinding behavior: Cognitive mapping and other spatial processes. Baltimore, MA: The Johns Hopkins University Press.

Hamid, S. N., Stankiewicz, B., \& Hayhoe, M. (2010). Gaze patterns in navigation: Encoding information in large-scale environments. Journal of Vision, 10, 1-11, http://www.journalofvision.org/content/10/12/28, doi:10.1167/10.12.28.

Harris, M. A., \& Wolbers, T. (2014). How age-related strategy switching deficits affect wayfinding in complex environments. Neurobiology of Aging, 35, 1095-1102.

Hazen, N. L., Lockman, J. L., and Pick, H. L. (1978). The development of children's representations of large-scale environments. Child Development, 49, 623-636. 
Hegarty, M., Montello, D. R., Richardson, A. E., Ishikawa, T., \& Lovelace, K. (2006). Spatial abilities at different scales: Individual differences in aptitude-test performance and spatial-layout learning. Intelligence, 34, 151-176.

Hegarty, M., Richardson, A. E., Montello, D. R., Lovelace, K., \& Subbiah, I. (2002). Development of a self-report measure of environmental spatial ability. Intelligence, 30, 425-447.

Hegarty, M., \& Waller, D. (2004). A dissociation between mental rotation and perspective-taking spatial abilities. Intelligence, 32, 175-191.

Ishikawa, T., \& Montello, D. R. (2006). Spatial knowledge acquisition from direct experience in the environment: Individual differences in the development of metric knowledge and the integration of separately learned places. Cognitive Psychology, 52, 93-129.

Klatzky, R. L., Loomis, J. M., Golledge, R. G., Cicinelli, J. G., Doherty, S., \& Pellegrino, J. W. (1990). Acquisition of route and survey knowledge in the absence of vision. Journal of Motor Behvavior, 22, 19-43.

Kozhevnikov, M., \& Hegarty, M. (2001). A dissociation between object manipulation spatial ability and spatial orientation ability. Memory \& Cognition, 29, 745-756.

Lahiri, U., Trewyn, A., Warren, Z., \& Sarkar, N. (2011). Dynamic eye gaze and its potential in virtual reality based applications for children with Autism Spectrum Disorders. Autism Open Access, 1, 10000101.

Landau, B., Spelke, E., \& Gleitman, H. (1984). Spatial knowledge in a young blind child. Cognition, 16, 225-260.

Loomis, J. M., Klatzky, R. L., Golledge, R. G., Cicinelli, J. G., Pellegrino, J. W., \& Fry, P. (1993). Nonvisual navigation by blind and sighted: Assessment of path integration ability. Journal of Experimental Psychology: General, 122, 73-91.

Montello, D. R. (1998). A new framework for understanding the acquisition of spatial knowledge in large-scale environments. In M. J. Egenhofer, \& R. G. Golledge (Eds.), Spatial and temporal reasoning in geographic information systems. (pp.143-154). Oxford: Oxford University Press.

Munn, S. M., Stefano, L., \& Pelz, J. B. (2008). Fixation-identification in dynamic scenes: Comparing an automated algorithm to manual coding. Proceedings of the $5^{\text {th }}$ Symposium on Applied Perception in Graphics and Visualization. Los Angeles, California.

Nazareth, A., Odean, R., \& Pruden, S. M. (2017). The Use of Eye-tracking in Spatial Thinking Research. In C. Was, F. Sansosti, \& B. Morris (Eds.), Eye-Tracking Technology Applications in Educational Research (pp. 239-260). Hershey, PA: IGI Global.

O’Keefe, J., \& Nadel, L. (1978). The Hippocampus as a Cognitive Map. Oxford: Oxford University Press. 
Ploran, E. J., Rovira, E., Thompson, J. C., \& Parasuraman, R. (2015). Underlying spatial skills to support navigation through large, unconstrained environments. Applied Cognitive Psychology, 29, 608-613.

Presson, C. C., \& Montello, D. R. (1988). Points of reference in spatial cognition: Stalking the elusive landmark. British Journal of Developmental Psychology, 6, 378-381.

Psychology Software Tools, Inc. [E-Prime 2.0]. (2012). Retrieved from http://www.pstnet.com.

Salvucci, D. D., \& Goldberg, J. H. (2000). Identifying fixations and saccades in eyetracking protocols. In Proceedings of the Eye Tracking Research and Applications Symposium (pp.71-78). New York: ACM Press.

Siegel, A. W., \& White, S. H. (1975). The development of large-scale environments. Advances in Child Development and Behaviour, 10, 9-55.

Snodgrass, J. G., \& Corwin, J. (1988). Pragmatics of measuring recognition memory: Applications to dementia and amnesia. Journal of Experimental Psychology: General, 117, 34-50.

Sorrows, M. E., \& Hirtle, S. C. (1999). The nature of landmarks for real and electronic spaces. In C. Freksa, D. M. Mark (Eds.), Spatial information theory: Cognitive and computational foundations of geographic information science, COSIT '99, Vol. 1661 Lecture Notes in Computer Science (pp. 37-50). Berlin: Springer.

Strayer, D. L., Drews, F. A., \& Johnston, W. A. (2003). Cell phone-induced failures of visual attention during simulated driving. Journal of Experimental Psychology: Applied, 9, 23-32.

Sutton, J. E., Buset, M, \& Keller, M. (2014). Navigation experience and mental representations of the environment: Do pilots build better cognitive maps? PLoS ONE, 9, e90058.

Sutton, J. E., Keller, M., \& Vollebregt, M. (2017). Navigating (in) the second decade of life: Cognitive maps improve across adolescence. Manuscript submitted for publication.

Sutton, J. E., Vollebregt, M., \& Grogan, B. (2016, November). Self-guided exploration of a novel environment results in a more accurate cognitive map than learning via route integration. Paper presented at the $57^{\text {th }}$ annual meeting of the Psychonomic Society, Boston, MA.

Tolman, E. C. (1948). Cognitive maps in rats and men. The Psychological Review, 55, 189-208.

Tolman, E. C., Ritchie, B. F., \& Kalish, D. (1946). Studies in spatial learning: I. Orientation and the short-cut. Journal of Experimental Psychology, 36, 13-24.

Waller, D., \& Greenauer, N. (2007). The role of body-based sensory information in the acquisition of enduring spatial representations. Psychological Research, 71, 322332. 
Weisberg, S. M., \& Newcombe, N. S. (2016). How do (some) people make a cognitive map? Routes, places, and working memory. Journal of Experimental Psychology: Learning, Memory, and Cognition, 42, 768-785.

Weisberg, S. M., Schinazi, V. R., Newcombe, N. S., Shipley, T. F., \& Epstein, R. A. (2014). Variations in cognitive maps: Understanding individual differences in navigation. Journal of Experimental Psychology: Learning, Memory, and Cognition, 40, 669-682.

Wen, W., Ishikawa, T., \& Sato, T. (2011). Working memory in spatial knowledge acquisition: Differences in encoding processes and sense of direction. Applied Cognitive Psychology, 25, 654-662.

Wen, W., Ishikawa, T., \& Sato, T. (2013). Individual differences in the encoding processes of egocentric and allocentric survey knowledge. Cognitive Science, 37, 176-192.

Wolbers, T., \& Hegarty, M. (2010). What determines our navigational abilities? Trends in Cognitive Sciences, 14, 138-146.

Yantis, S. (1993). Stimulus-driven attentional capture and attentional control settings. Journal of Experimental Psychology: Human Perception and Performance, 19, 676-681.

Yantis, S, \& Jonides, J. (1990). Abrupt visual onsets and selective attention: Voluntary versus automatic allocation. Journal of Experimental Psychology: Human Perception and Performance, 16, 121-134. 


\section{Appendix A: Ethics Approval}

Western

Research

\section{Western University Non-Medical Research Ethics Board \\ NMREB Delegated Initial Approval Notice}

Principal Investigator: Dr. Jennifer Sutton

Department \& Institution: BrescialPsychology (BUC), Western University

NMREB File Number: 107907

Study Title: Investigating individual differences in navigation using eyetracking

Sponsor: NSERC: Navigation and Cognitive Maps

NMREB Initial Approval Date: April 21, 2016

NMREB Expiry Date: April 21, 2017

Documents Approved and/or Received for Information:

\begin{tabular}{|l|l|l|}
\hline Document Name & Comments & $\begin{array}{l}\text { Version } \\
\text { Date }\end{array}$ \\
\hline $\begin{array}{l}\text { Letter of Information \& } \\
\text { Consent }\end{array}$ & LOI and Consent & $2016 / 03 / 24$ \\
\hline Recruitment Items & SONA/Participant Pool Recruitment & $2016 / 03 / 24$ \\
\hline Instruments & Demographic Questionnaire & $2016 / 03 / 24$ \\
\hline Recruitment Items & Recruitment Poster & $2016 / 04 / 19$ \\
\hline Western University Protocol & & $2016 / 04 / 15$ \\
\hline Instruments & Practice for Direction Estimation Task & $2016 / 03 / 23$ \\
\hline Instruments & Route Construction Task - Received March 23, 2016 & \\
\hline Instruments & Santa Barbara Sense of Direction Scale (SBSOD) - Received March 23, & \\
\hline Instruments & 2016 & \\
\hline Instruments & Silcton Buildings List - Received March 23, 2016 & \\
\hline Instruments & Spatial Orientation Test (SOT) - Received March 23, 2016 & \\
\hline Instruments & Direction Estimation Task - Received March 23, 2016 & \\
\hline Instruments & Landmark Recognition Task - Received March 23, 2016 & \\
\hline & Model Building Task - Received March 23, 2016 & \\
\hline
\end{tabular}

The Western University Non-Medical Research Ethics Board (NMREB) has reviewed and approved the above named study, as of the NMREB Initial Approval Date noted above.

NMREB approval for this study remains valid until the NMREB Expiry Date noted above, conditional to timely submission and acceptance of NMREB Continuing Ethics Review.

The Western University NMREB operates in compliance with the Tri-Council Policy Statement Ethical Conduct for Research Involving Humans (TCPS2), the Ontario Personal Health Information Protection Act (PHIPA, 2004), and the applicable laws and regulations of Ontario.

Members of the NMREB who are named as Investigators in research studies do not participate in discussions related to, nor vote on such studies when they are presented to the REB.

The NMREB is registered with the U.S. Department of Health \& Human Services under the IRB registration number IRB 00000941:

Ethics Officer, on behalf of Dr. Riley Hinson, NMREB Chair or delegated board member

Ethics Officer to Contact for Further Information: Erika Basile Nicole Kaniki Grace Kelly Katelyn Harris L Vikki Tran

Western University, Research, Support Services BIdg.. Ste. 5150

London, ON. Canada N6G 1 G9 t. 519.661.2161 f. 519.661.3907 www.westernu.ca/research 


\section{Curriculum Vitae}

Name: Mikayla Keller

\section{Post-secondary Education and Degrees:}

Brescia University College; The University of Western Ontario London, Ontario, Canada

$2010-2014$

B.A. Psychology, Honours Specialization

The University of Western Ontario

London, Ontario, Canada

2015 - Present

M.Sc. Psychology, Cognition

\section{Honours and Awards:}

Academic Dean's Entrance Scholarship

2010 - 2011, 2011 - 2012, 2012 - 2013, 2013 - 2014

Western Graduate Research Scholarship

2015 - Present

Stanley Nelson Roscoe Best Student Paper Award - Finalist and Second Runner-up - $19^{\text {th }}$ International Symposium on Aviation Psychology 2017

\section{Related Work Experience:}

Research Assistant to Dr. Jennifer Sutton

Spatial Cognition Laboratory, Department of Psychology

Brescia University College

March 2013 - September 2015

Teaching Assistant

The University of Western Ontario

September 2015 - Present

\section{Publications:}

Sutton, J. E., Buset, M., \& Keller, M. (2014). Navigation experience and mental representations of the environment: Do pilots build better cognitive maps? PLoS ONE, 9, e90058. doi:10.1371/journal.pone.0090058 
Keller, M., \& Sutton, J. E. (2017). Flight experience and mental representations of space. In Proceedings of the $19^{\text {th }}$ International Symposium on Aviation Psychology (pp. 256 - 261). Dayton, OH: Association of Aviation Psychology.

Sutton, J. E., Keller, M., \& Vollebregt, M. (2017). Navigating (in) the second decade of life: Cognitive maps improve across adolescence. Manuscript submitted for publication. 